\title{
Fault Tolerance in Wireless Sensor Network
}

\author{
Samuel Devakumar P.V ${ }^{1}$, Mr.N.Ilayaraja ${ }^{2}$ \\ Post Graduate Student, Department of Computer Sciences and Engineering, \\ R.V.S Technical Campus, Coimbatore, $\operatorname{India}^{1}$ \\ Assistant Professor, Department of Computer Sciences and Engineering, \\ R.V.S Technical Campus, Coimbatore, India ${ }^{2}$
}

\begin{abstract}
Wireless sensors which are used for sensing any event can be made intelligent by adding cognitive sensors. Cognitive agents are capable of making proactive choices according to the current situation based on the requirement needed in a WSN, to achieve the end to end goals of the network deployed even during the complex situation and with constraints scenario. The cognitive layer can be added to any network deployed in any application environment and it can be made intelligent. The cognitive sensor is a recent technology, and this literature survey reviews how cognitive layer has been implemented in various applications. Energy loss is one of the major impediments in all WSNs. This literature survey shows how energy efficiency can be minimized.
\end{abstract}

Keywords: Cognitive Sensor, Energy Efficient, Reliable, Cost - effective

\section{INTRODUCTION}

WSN's are contiguous autonomous sensors generally deployed to monitor physical or environmental conditions while passing the data via the network to the target location. Nodes are the base for any WSN and it can range from few to several thousand and the topology also varies from a simple star to advanced multi-hop mesh networks. Propagation technique between the nodes is referred as routing or flooding. The applications for such networks span across various fields like area monitoring (geo-fencing), Healthcare monitoring (body-area networks) and environmental sensing (forest fire or landslide detection) and across industries for highly complicated procedures such as machine health monitoring and data logging. Cognitive Wireless Sensor Networks (CWSN) is a technology that provides effective utilization of the spectrum by inducing cognitive abilities to the sensors which in turn lets them change and adapt their communication parameters according to the changes in their environment. The major difference between the former and latter is that while WSN has fixed communication challenges, CWSN has no fixed channels and utilizes the vacant ones or channels.

\section{COGNITIVE SENSOR NETWORKS}

Cognitive networks were first proposed by Theo Kantor in June 1998 where he defined CN as a network with memory and a network that can clearly distinguish natural environments that don't match the models and declare errors, later IBM provided the foundation of Cognitive Wireless Networks which was theoretically explained as self-organizing and self-aware networks that could perceive conditions, plan, decide and act on it.

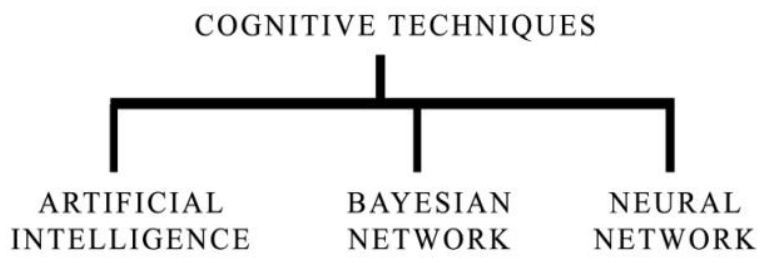

A. Artificial Intelligence

Artificial Intelligence can be simply put in words as intelligent behavior by machines rather than the natural intelligence possessed by humans and animals, or it could be briefed as perceiving the environmental behavior and act accordingly to achieve its goals. The scope for AI is evenly distributed among various industries from agriculture to networking. Artificial intelligence enabled networks are able to predict traffic better by collecting and analyzing real time data and boasts a lot of fool proof methods that can anticipate and defend itself against malicious activities like DDoS attacks and hack attempts. 


\title{
International Journal of Advanced Research in Computer and Communication Engineering
}

\author{
Vol. 8, Issue 4, April 2019
}

\section{B. Bayesian Network}

Bayesian Network is also referred to as a probabilistic directed a cyclical model, and generally, it is used to represent the conditional dependency between random variables through a Directed Acyclic Graph (DAG). Two main components in the Bayesian Networks include nodes and edges where the nodes represent the random variables and the edges show the relationship between them. Dynamic Bayesian Networks can be implemented in to adapt with real-time monitoring.

\section{Neural Networks}

Neural Networks simply means the biological neural network. Artificial Neural Networks (ANN) is inspired by the native biological neural networks. It is an interconnected group of nodes like the neurons in the brain and similarly, here we've nodes and arrows where the nodes represent artificial neurons and arrows represent the connection between them which also acts as a medium of communication between these nodes. Implementing ANN in CRN can improve the learning and decision-making capacity without affecting any important parameters.

\section{LITERATURE SURVEY}

In this survey, we explain how Bayesian Network could be implemented to make the network cognitive and improving the fault tolerance.

\section{A. Cognitive Sensor}

Gayathri Vijay et al. in the paper provides a comparative study of the different cognitive techniques applied to wireless sensor network applications in recent times, and evaluating their performance in achieving the network's objective. Cognition means the process of learning through perception, reasoning, and knowledge. When the nodes with cognitive capabilities are introduced into an entire network of communicating sensor nodes, it gives chance to exciting new opportunities in wireless sensor network research that could overcome the limitations in the current design technique. A cognitive network is said to be adaptive, self-aware and self-organizing network capable of adapting itself based on observing, learning and sharing information. It also requires a SAN (Software Adaptable Framework) to implement the goals of the cognitive network. This paper also discusses the current trends in WSN research, which includes various cognitive techniques adopted by researchers to improve the performance of WSNs. The advantages of using cognitive approaches lead to improved performance of the network and making it more reliable. The challenge that is being faced is deciding on the optimal Deployment architecture of the cognitive capability enhanced nodes is also a challenging problem.

Ryan W. Thomas et al. focuses on the future research and foundation of Cognitive Networks $(\mathrm{CN})$. CNs is motivated by reliability, complexity in a network. They have compared cognitive network with Cognitive Radio (CR) and crosslayer design. Current network technology limits the network ability to adapt. Cognitive networks remove limitation allowing the network to observe study and act according to the scenario. Cognitive network gives a new hope and approach to dealing with the complexity and possible application difference in cognitive network compared to cognitive radio and cross-layer design. A cognitive network requires SAN to implement actual network flow functionality and allow the cognitive process to adapt the network. Cognitive process is the heart of the performance optimization. A CR can be implemented only on the wireless network where $\mathrm{CN}$ can be implemented both on wired and wireless medium. This paper gives an idea of why and what for cognitive networks are used and how complex issues can be rectified. Implementation of a $\mathrm{CN}$ in real-time and making it reliable might be slightly complex as it has to change its configuration regularly depending on the need of the network. A significant amount of work remains to be done to make cognitive networks a reality, the rising complexity of networks and the need to manage this complexity makes the concept timely and attractive.

Anuroop Gaddam et al. focuses on developing a smart home monitoring system using intelligent wireless sensor system for helping the elderly people as the number of elderly people increases day by day, this paper has developed a solution for the elderly people to provide safe and secure environment especially when living alone at home. The system consists of many cognitive sensors that are actively sensing the user activity. A profile is being built and daily activities are monitored and stored in the profile, any abnormal activity in the users' behavior will trigger an alert message to the caretaker or the family member. This system is implemented using minimum number of optimal cognitive sensor close to the potential problem source instead of deploying huge number of wireless sensors. The advantages in this system include coverage, ease of deployment, low energy consumption, reduced on maintenance and more acceptability. The system can be installed in any residential environment without any complexity.

This can also be easily installed in an existing home environment with no major modifications or damage. A lot more features are planned to be added to the system in the future and can be incorporated in the sensor itself, as the sensor itself qualifies as a cognitive sensor. 


\author{
Vol. 8, Issue 4, April 2019
}

Michele Chincoli et al. show that interference is one of the major issues in a WSN that limits reliability and QoS guarantees. Interference is caused due to the air medium around the network created by $\mathrm{Bluetooth}$ and $\mathrm{Wi}-\mathrm{Fi}$ technologies, which make the network weak, to avoid this flaw, interference must be avoided to make the network reliable for communication. The reliability of WSNs becomes a complex problem, due to unpredictable interference and fading in the wireless shared medium. IEEE standard has come up with an upgraded version of the standard, 802.15.4e, where the wireless sensor nodes can access the medium by Time Division Multiple Access (TDMA) and channel hopping. They propose an algorithm called Multiclass Feed forward Neural Network (MFNN) which can predict the channel and can provide high performance such as low latency and maximum throughput at any given time slot. One of the advantages of MFNN is even in minimum power sensor networks, the algorithm could be deployed in a distributed manner. This research will serve as a base for future studies, considering cognitive technique with real-time data consumption.

Waleed Youssef et al. proposed that in WSN the data that are gathered by the nodes are gathered and sent to the gateway for processing and forwarded to the remote command centre. There are possible threats to the gateway to dangerous events such as e.g. fire, which damage thus making the network not functioning. Moving the gateway to a secure zone is critical for the operation of the network. Also, moving the gateway too far from the network may decrease the performance of the network such as throughput and energy consumption. Balancing the gateway safety and network performance is important. To achieve this goal GRISP algorithm, a novel Gateway Relocation algorithm is used. GRISP uses an evolutionary neural network model to evaluate the safety of the gateway at the various locations. The model is used to search the area for a safer position that would enhance or maintain an acceptable level of network performance. GRISP also guides the gateway during the move by finding safe paths leading to the new location. The main aim is to keep the gateway out of harm and to keep the network fully operational while the gateway is in transit.

The Advantages of GRISP are, it selects the best location in safety and performance for the gateway. GRISP ensures that the path with the best performance and safety measures is selected. Experiment results have proved that GRISP was able to protect the gateway by moving to secure positions in the environments and also made sure that the network is operational and safe.

\title{
B. Bayesian Optimization Algorithms
}

Bhaskar Krishnamachari et al. try to propose the first solution in handling a fault-event disambiguation problem that frequently occurs in sensors. They suggest implementing a distributed solution in wireless sensor networks for canonical tasks by binarily detecting the environmental events. By implementing Distributed Bayesian algorithm on sensor measurement faults, they tend to detect and correct them effectively. Correlation between faulty sensor events is examined from neighboring sensors to rule out disambiguate data, but sometimes this may introduce new errors if the surrounding sensors give faulty data. Initially, they proposed two Bayesian algorithms namely Randomized decision scheme and threshold decision scheme but later decided to stick with threshold scheme as the latter one outperforms the former in minimizing errors. Simulations have proven that even if the $10 \%$ of the total nodes are faulty this technique can achieve $85-95 \%$ of fault correction

Behnaz Moradabadi et al. propose that the estimation of distribution algorithms have emerged as a technique for estimating population distribution in evolutionary algorithms. The Bayesian optimization algorithm is a calculation of distribution algorithm, which uses a Bayesian network to calculate the candidate distribution and to create the next sample generation form the previously constructed network. The results of the experiment show that the Bayesian optimization algorithm is capable of finding the link between the variables of the optimization problems. Bayesian network is built using a typical Bayesian optimization algorithm that uses greedy algorithm. This paper proposes a contemporary Bayesian algorithm to solve continuous optimization problems employing learning automata for building the Bayesian network. During the execution of the algorithm, the group of learning automata tries to learn the optimal Bayesian network structure. The use of this method advances an algorithm which helps in building the Bayesian network in lesser computation time.

Experiments results obtained show the effect of the proposed algorithm on the optimization problems.

Wiboonsak Watthayu et al. explains Multi-criteria decision making (MCDM) as a process which incorporates actions such as selecting the best of all possible alternatives by evaluating each against the other, focusing on conflicts. Since the majority of the models had uncertain interactions that weren't coherent or systematic, they proposed a framework based on Influence diagram and Bayesian networks. ID's are used to represent decision nodes with all possible alternatives and utility nodes comprising of decision criteria and the factors that may affect them. Links are used in qualitatively modeling the interdependencies and Conditional probability tables (CPT) are used in quantitative 


\title{
International Journal of Advanced Research in Computer and Communication Engineering
}

\author{
Vol. 8, Issue 4, April 2019
}

association of chance nodes and utility nodes. The variable's inter-dependency knowledge of the domain expert is measured using the joint probability distribution. The requirements of the decision maker are used. Any of the Bayesian Network or the Influence diagram algorithms can be deployed to derive an effective solution. Specifically, employing Bayesian network in an MCDM problem allows to deriving a probabilistic interdependency among the elements involved. This framework is an effective modeling tool for making MCDM based decisions under uncertain environments.

S. Gheisari et al. 2016 proposes an approach to solve the major problem faced in finding the optimal network structure using the available data. Mostly greedy algorithms are used in solving the class of NP-Hard problems. Two novel learning automata-based algorithms are used to solve the Bayesian networks structure learning problems which use the edges to calculate direction and appearance of a network. The game with common payoff and competitive game are the two special cases mentioned in this paper where all automata receive a unique payoff in the former one and each one receiving its own in the latter and over time we switch to the BN's with higher scores. Experiments based on BIC and Hamming distance were conducted to evaluate the performances of LA-CP and LA-DP with the proposed approach. Although LA-CP performs better than the other classifiers on average results prove that comparing with other searchbased methods the newly proposed algorithms easily outperform them by coming up with optimal structure in a nominal time.

\section{Fault Tolerance}

Yibing Zou et al. briefs on how Cognitive Radio Technology (CRN) was introduced by Cognitive Sensor Networks (CSN) to improve the spectrum efficiency by spectrum sensing. But spectrum sensing requires more energy which heavily decreases the lifetime of the network, so they proposed a new sensing scheme based on the cooperative spectrum to select the sensing node for CSN with energy harvesting. The problem is formulated as an NP-hard 0-1 integer programming problem and to solve the same they map the $0-1$ variables into continues to come up with an equivalent convex optimization problem. To reduce the complexity a priority based sensing nodes is employed and based on these priorities the optimal sensing node is determined. Energy causality constraint and collision constraint are the main factors to be considered before choosing the sleep scheduling algorithms proposed algorithms have been proven efficacious in balancing the network energy consumption while prolonging the lifetime of the network. .

Maryam Najimi et al. proves that by focusing on node selection criteria for spectrum sensing it's possible to maximize the lifetime of Cognitive Sensor Networks(CSN). This can be achieved by setting constraints on the performance of sensors which sense the spectrum. The problem has to be simplified to a more pliable form since the native begins as an NP-complete problem which has no standard methods to solve it. Optimal conditions are obtained by deploying convex optimization frameworks which detect the sensors that sense the spectrum. Although the algorithm doesn't. Minimize the energy consumption it balances the sensor's residual energy over time simulating with similar algorithms and evaluating the results the proposed algorithm proves itself to be efficient in maximizing the network's lifetime.

Ali Valehi et al. proposes a joint framing and scheduling policy that optimizes the energy efficiency to manage a cognitive wireless sensor that transmits and measures samples to a common sink node, which are made possible by the channel vacancies of a primary network. It mainly focuses on improving the age of information which is defined as the time taken from sampling epoch to the successful delivery of sample taking framing, queuing and waiting time of the channel vacancies. A number based framing-policy that determines the samples in data packets with the same header sizes are proposed. By characterizing the utilized queue dynamics, packet discards rate and retransmission probability a low cost and energy efficient network of unlicensed sensors comprising of delay-sensitive applications such as body area sensor networks is developed. Since the channel variation is much slower comparing with a single packet transmission rate this can be used in framing an effective policy for time-varying shared channels.

Meng Zheng et al. explains the optimization of spectrum sensing for an effective access to cognitive sensor networks, though it is extremely energy consuming this paper effectively employs CoMAC based cooperative spectrum sensing for estimating the presence of the primary users. Energy efficiency is an important performance metric for CSN's. This technique focuses on optimising the non-convex and non-separable problems by finding an expression for detection threshold and proposes an iterative algorithm which solves two sub problems, mainly the sequence length optimization and sensing time optimization. The optimality of the algorithm is evaluated through simulations thereby confirming that a combination of CoMAC-based CSS and the algorithm proposed produces much higher EE than the conventional techniques without any compromise in the performance. This confirms the quasi-convexity by usage of bi-section based algorithms for a sensing time optimization problem for a given length of the symbol sequence. 


\title{
International Journal of Advanced Research in Computer and Communication Engineering
}

\author{
Vol. 8, Issue 4, April 2019
}

\section{CONCLUSION}

There is an ongoing demand on CWSN for adapting the various changes in the environment. This has been discussed briefly above along with the techniques to implement it. The study shows that predicting the faults in the network beforehand using cognitive techniques will improve the performance of the network.

\section{REFERENCES}

[1]. Thomas, R., Friend, D., Dasilva, L. and Mackenzie, A. (2006). Cognitive networks: adaptation and learning to achieve end-to-end performance objectives. IEEE Communications Magazine, 44(12), pp.51-57.

[2]. Gaddam, A., Mukhopadhyay, S. and Gupta, G. (2011). Elder Care Based on Cognitive Sensor Network. IEEE Sensors Journal, 11(3), pp.574581 .

[3]. G. Vijay, E. Bdira, and M. Ibnkahla, "Cognitive approaches in Wireless Sensor Networks: A survey," 201025 th Biennial Symposium on Communications, 2010.

[4]. M. Chincoli, P. D. Boef, and A. Liotta, "Cognitive channel selection for Wireless Sensor communications," 2017 IEEE 14th International Conference on Networking, Sensing and Control (ICNSC), 2017.

[5]. Youssef, W. and Younis, M. (2007). A cognitive scheme for gateway protection in wireless sensor network. Applied Intelligence, 29(3), pp.216-227.

[6]. Moradabadi, B., Ebadzadeh, M. and Meybodi, M. (2016). A new real-coded stochastic Bayesian optimization algorithm for continuous global optimization. Genetic Programming and Evolvable Machines, 17(2), pp.145-167.

[7]. W. Watthayu, Y. Peng, A Bayesian network based framework for multi criteria decision making, in: Proceedings of the 17th International Conference on Multiple Criteria Decision Analysis, 2004.

[8]. Gheisari, S., Meybodi, M., Dehghan, M. and Ebadzadeh, M. (2016). Bayesian network structure training based on a game of learning automata. International Journal of Machine Learning and Cybernetics, 8(4), pp.1093-1105.

[9]. Meng Zheng, Lin Chen, Wei Liang, Haibin Yu, and Jinsong Wu, Energy-Efficiency Maximization for Cooperative Spectrum Sensing in Cognitive Sensor Networks, IEEE Transaction on green communications and networking, March 2017.

[10]. Ali Valehi, Abolfazl Razi, Maximizing Energy Efficiency of Cognitive Wireless Sensor Networks with Constrained Age of Information, IEEE transaction on Cognitive Communication and networking 2017

[11]. Y. Zou, J. Peng, K. Liu, F. Jiang, and H. Lu, "Energy-efficient cooperative spectrum sensing for cognitive sensor networks with energy harvesting," 2016 Chinese Control and Decision Conference (CCDC), 2016.

[12]. Najimi, M., Ebrahimzadeh, A., Andargoli, S. and Fallahi, A. (2014). Lifetime Maximization in Cognitive Sensor Networks Based on the Node Selection. IEEE Sensors Journal, 14(7), pp.2376-2383.

[13]. Krishnamachari, B. and Iyengar, S. (2004). Distributed Bayesian algorithms for fault-tolerant event region detection in wireless sensor networks. IEEE Transactions on Computers, 53(3), pp.241-250. 